\title{
Mikheyev-Smirnov-Wolfenstein enhancement of oscillations as a possible solution to the solar-neutrino problem
}

\author{
S. P. Rosen and J. M. Gelb \\ Theoretical Division, Los Alamos National Laboratory, Los Alamos, New Mexico 87545
}

(Received 7 April 1986)

\begin{abstract}
Mikheyev and Smirnov have observed that neutrino oscillations in the Sun can be greatly enhanced through the mechanism of Wolfenstein matter oscillations. We develop a qualitative understanding of this phenomenon in the small-mixing-angle limit and carry out extensive calculations in order to apply it to the solar-neutrino problem. Our simple theoretical model agrees remarkably well with the calculations. After determining those values of $\Delta m^{2}$ and $\sin ^{2} 2 \theta$ in the small-mixing-angle limit for which the ${ }^{8} \mathrm{~B}$ plus ${ }^{7} \mathrm{Be}$ neutrino capture rate in ${ }^{37} \mathrm{Cl}$ is suppressed by a factor $2-4$, we predict the corresponding capture rate for $p p$ plus ${ }^{7} \mathrm{Be}$ neutrinos in ${ }^{71} \mathrm{Ga}$. The gallium capture rate can range from no reduction to a factor of 10 reduction. We also determine the modified spectrum of ${ }^{8} \mathrm{~B}$ neutrinos arriving at Earth and discuss the importance of this spectrum as a means of choosing between oscillations and the solar model as the cause of the solar-neutrino problem, and also as a means of distinguishing between different sets of oscillation parameters.
\end{abstract}

\section{INTRODUCTION}

Mikheyev and Smirnov ${ }^{1}$ have recently observed that, given the high densities of matter encountered in the interior regions of the Sun, the oscillations between neutrino flavors caused by small mass differences can be greatly enhanced by Wolfenstein matter oscillations. ${ }^{2}$ In particular, intrinsically small mixing angles can be converted by this enhancement mechanism into large effective mixing angles, and a neutrino born as an electron neutrino $v_{e}$ in the core of the Sun can emerge from it as an almost pure muon neutrino $v_{\mu}$. This means that instead of regarding the solar-neutrino problem as a probe of oscillations with very small mass differences $\left(\Delta m^{2} \simeq 10^{-11} \mathrm{eV}^{2}\right)$, and relatively large mixing angles $\left(\sin ^{2} 2 \theta \simeq 0.3\right)$, we can also use it to explore very small mixing angles $\left(\sin ^{2} 2 \theta \simeq 0.001-0.1\right)$ and somewhat larger, but still terrestrially inaccessible, mass differences in the range of $10^{-8}-10^{-4} \mathrm{eV}^{2}$.

In this paper we describe a simplified model which explains the principal features of the Mikheyev-SmirnovWolfenstein (MSW) enhancement mechanism, and we report on a series of calculations in which it is assumed to be the cause of the reduction in the capture rate of ${ }^{8} \mathrm{~B}$ plus ${ }^{7} \mathrm{Be}$ (upper energy line) neutrinos in ${ }^{37} \mathrm{Cl}$ by a factor $2-4$, as observed by Davis. ${ }^{3}$ We locate that region in the $\left(\Delta m^{2}, \sin ^{2} 2 \theta\right)$ diagram which yields the requisite reduction in the Davis experiment, and we then predict the capture rate for $p p$ plus ${ }^{7} \mathrm{Be}$ (both energy lines) neutrinos in ${ }^{71} \mathrm{Ga}$ for each point in this region. We also calculate the corresponding modified spectra of ${ }^{8} \mathrm{~B}$ neutrinos arriving at Earth.

\section{OSCILLATIONS AND THE SOLAR MODEL}

On the basis of our calculations we can develop the following scenario for the solar-neutrino problem. There are two lines in the small- $\sin ^{2} 2 \theta$ region of the $\left(\Delta m^{2}, \sin ^{2} 2 \theta\right)$ plane for which the ${ }^{8} \mathrm{~B}$ plus ${ }^{7} \mathrm{Be}$ capture rate in ${ }^{37} \mathrm{Cl}$ is reduced by a factor $\simeq 3$ [see Fig. 5(a)]: (i) $\Delta m^{2} \simeq 10^{-4}$ for all $\sin ^{2} 2 \theta$ and (ii) $\log _{10}\left(\sin ^{2} 2 \theta\right)+\log _{10}\left(\Delta m^{2}\right) \simeq-7.5$ [Fig. $5(a)$ is a log-log plot.]

From calculations of the modified spectra of ${ }^{8} \mathrm{~B}$ neutrinos arriving at Earth [Figs. 6(a) through 6(f) and Table II], we see that the first line corresponds to the case in which the higher-energy ${ }^{8} \mathbf{B}$ neutrinos are suppressed, while the lower-energy ones are unaffected. (The separation between "high" and "low" occurs in the neighborhood of 5-7 MeV, depending on the value of $\sin ^{2} 2 \theta$.) By contrast, the second line corresponds to a suppression of all ${ }^{8} \mathbf{B}$ neutrinos, but most especially in the low- and middle-energy range. It follows that for oscillation parameters on the first line, the spectra of $p p$ plus ${ }^{7} \mathrm{Be}$ neutrinos will be unaltered, and the capture rate in ${ }^{71} \mathrm{Ga}$ will be exactly as in the standard model, namely, about 105 solar neutrino units ${ }^{4}$ (SNU). Parameters on the second line, however, modify the $p p$ and ${ }^{7} \mathrm{Be}$ spectra to varying degrees, and they can give reductions in the ${ }^{71} \mathrm{Ga}$ capture rate by as much as a factor of 10 . The calculated capture rates as functions of $\Delta m^{2}$ and $\sin ^{2} 2 \theta$ are shown in Table I.

The impact of the first-line solution on ${ }^{71} \mathrm{Ga}$ can also be understood on the basis of the spatial distribution of neutrino production. In Sec. IV we demonstrate that the first line corresponds to the occurrence of the MSW effect in the core of the Sun, and that the second line corresponds to the effect occurring in the main body of the Sun. A much higher percentage of ${ }^{8} \mathbf{B}$ neutrinos is created in the core than is the case for $p p$ or ${ }^{7} \mathrm{Be}$ neutrinos. ${ }^{4}$

The MSW mechanism is a beautiful device for enhancing oscillations with intrinsically small mixing angles, and it presents us with a most attractive solution to the solarneutrino puzzle. From this point of view, the ${ }^{71} \mathrm{Ga}$ experiment will enable us to determine whether the first or the second line is the correct solution, and a measurement of 
TABLE I. Various expected capture rates for $p p+{ }^{7} \mathrm{Be}$ in ${ }^{71} \mathrm{Ga}$ in SNU for parameters that yield a factor of 3 reduction in ${ }^{8} \mathrm{~B}+{ }^{7} \mathrm{Be}$ in ${ }^{37} \mathrm{Cl}$ (underlined values). The rest were filled in for completeness. $100 \mathrm{SNU}$ was taken to be $100 \%$.

\begin{tabular}{ccccccc}
\hline \hline$\Delta m^{2} \mathrm{eV}^{2} \sin ^{2} 2 \theta$ & $10^{-3.0}$ & $10^{-2.5}$ & $10^{-2.0}$ & $10^{-1.5}$ & $10^{-1.0}$ & $10^{-0.5}$ \\
\hline $1.1 \times 10^{-4}$ & 100 & $\underline{100}$ & 100 & 100 & 100 & 100 \\
$1.0 \times 10^{-4}$ & $\underline{100}$ & 100 & $\underline{100}$ & $\underline{100}$ & 100 & 100 \\
$9.5 \times 10^{-5}$ & 100 & 100 & 100 & 100 & $\underline{100}$ & 100 \\
$5.8 \times 10^{-5}$ & $\underline{100}$ & 100 & 100 & 100 & 100 & 100 \\
$5.0 \times 10^{-5}$ & 100 & 100 & 100 & 100 & 100 & $\underline{100}$ \\
$1.7 \times 10^{-5}$ & 100 & $\underline{100}$ & 100 & 100 & 100 & 95 \\
$3.6 \times 10^{-6}$ & 65 & 60 & $\underline{60}$ & 60 & 50 & 45 \\
$1.1 \times 10^{-6}$ & 45 & 15 & 10 & $\underline{10}$ & 20 & 25 \\
$3.5 \times 10^{-7}$ & 70 & 40 & 10 & 5 & $\underline{5}$ & 20 \\
$1.0 \times 10^{-7}$ & 85 & 70 & 40 & 10 & 5 & $\underline{20}$ \\
\hline \hline
\end{tabular}

the spectrum will provide the final confirmation for it. The spectrum can also be used to remove ambiguities between the MSW mechanism and older attempts to solve the puzzle.

For example, should the outcome of the ${ }^{71} \mathrm{Ga}$ experiment be exactly as predicted by the standard solar model, then the first-line MSW solution will most likely be correct. However, unless we measure the spectrum of $v_{e}$ from ${ }^{8} \mathrm{~B}$ decay arriving at Earth, we will not be able to exclude some modification of the solar model as an explanation of the Davis experiment. The first-line solution leads to a suppression of the higher-energy half of the spectrum, whereas a modification of the solar model will reduce the overall normalization, but not the shape of the spectrum.

Should the outcome of the ${ }^{71} \mathrm{Ga}$ experiment be a significantly reduced capture rate, as compared with the predictions of the standard solar model, then we can certainly conclude that neutrino oscillations are taking place. In the event that the reduction factor for ${ }^{71} \mathrm{Ga}$ turns out to be smaller than that for ${ }^{37} \mathrm{Cl}$ (say, $\frac{3}{2}$ instead of 3) or much larger (say, 5-10, see Table I), it would be most likely that the second-line MSW solution is correct. If however, the reduction factors for the two experiments are approximately equal to one another, then we will have to choose between MSW-enhanced oscillations with parameters in the range $\sin ^{2} 2 \theta \simeq 10^{-2}-10^{-1.5}$ and $\Delta m^{2} \simeq 4-1 \times 10^{-6}$ $\mathrm{eV}^{2}$ (see Table I), and the possibility of large mixing angles $\left(\sin ^{2} 2 \theta \simeq 0.3-0.4\right)$ with small $\Delta m^{2}\left(\simeq 10^{-10}-10^{-12}\right.$ $\mathrm{eV}^{2}$ ). Again we can use the spectrum to make the choice.

If we plot the probability that a $v_{e}$ emitted in an ${ }^{8} \mathrm{~B}$ decay in the Sun remains a $v_{e}$ when it arrives at Earth, as a function of energy, i.e., $P\left(v_{e} \rightarrow v_{e} ; E_{v}\right)$, then we find that for oscillation parameters in the MSW range, the lowerenergy neutrinos are heavily suppressed, but the probability function climbs steadily to a value of $0.4-0.5$ at the high-energy end [see Figs. 6(c) and 6(d)]. In the case of large mixing angles and $\Delta m^{2} \simeq 10^{-10} \mathrm{eV}^{2}$, the oscillation lengths for both ${ }^{8} \mathbf{B}$ and $p p$ neutrinos are much smaller than the astronomical unit, and so the suppression is independent of energy ( $\left.P \simeq 1-\frac{1}{2} \sin ^{2} 2 \theta\right)$. As $\Delta m^{2}$ becomes smaller, some oscillatory structure will develop in
$P\left(v_{e} \rightarrow v_{e} ; E_{v}\right)$ for ${ }^{8} \mathrm{~B}$ neutrinos, but at the high-energy end the probability will generally tend to a value close to unity.

\section{SLAB MODEL}

We now describe a simple model for the MSW effect which accounts for most of the qualitative features, and even the quantitative features, of our computations in the case of small mixing angles.

According to Wolfenstein ${ }^{2}$ the time development of vacuum and matter oscillations is described by the differential equation

$$
i \frac{d}{d t}\left(\begin{array}{l}
v_{e} \\
v_{\mu}
\end{array}\right)=\left(\begin{array}{ll}
A & B \\
B & D
\end{array}\right)\left(\begin{array}{l}
v_{e} \\
v_{\mu}
\end{array}\right)
$$

in the basis of eigenstates $\left|v_{e}\right\rangle$ and $\left|v_{\mu}\right\rangle$. The elements of the "Hamiltonian" matrix are

$$
\begin{aligned}
& A \equiv \frac{m_{1}{ }^{2} c^{2}+m_{2}{ }^{2} s^{2}}{2 E}+\sqrt{2} G_{F} N_{e}, \\
& B \equiv \frac{\Delta m^{2}}{2 E} c s, \\
& D \equiv \frac{m_{1}{ }^{2} s^{2}+m_{2}{ }^{2} c^{2}}{2 E},
\end{aligned}
$$

where $m_{1}$ and $m_{2}$ are the masses of the neutrino mass eigenstates $\left|v_{1}\right\rangle$ and $\left|v_{2}\right\rangle$, respectively, and $\Delta m^{2}$ $\equiv m_{2}{ }^{2}-m_{1}{ }^{2}$ is measured in units of $\mathrm{eV}^{2}$. The mixing angle $\theta$ is defined by $c \equiv \cos \theta, s \equiv \sin \theta$, and

$$
\begin{aligned}
& \left|v_{e}\right\rangle=c\left|v_{1}\right\rangle+s\left|v_{2}\right\rangle, \\
& \left|v_{\mu}\right\rangle=-s\left|v_{1}\right\rangle+c\left|v_{2}\right\rangle .
\end{aligned}
$$

$G_{F}$ is the Fermi constant, $N_{e}$ is the density of electrons in the Sun, and $E$ is the neutrino energy (momentum) measured in units of $\mathrm{MeV}$. The location of the neutrino in the Sun at any time $t$ is $R$, where $R \simeq c t=t(\hbar=c \equiv 1)$.

It should be noted that the matter oscillation contribution to $A$, namely, $\sqrt{2} G_{F} N_{e}$ in (3.2), differs from that originally given by Wolfenstein in the factor of $\sqrt{2}$ and in 
sign. The factor of $\sqrt{2}$ has been noted by several authors 5 and it comes about because, when one evaluates the coherent (i.e., spin-nonflip) part of the $W^{+}$-exchange diagram for $v_{e}-e$ scattering in the electron rest frame, one must keep in mind that the incident neutrino is polarized, essentially $100 \%$. As for the sign, there are now three independent arguments which agree with the one above: one is a direct calculation by Langacker; ${ }^{6}$ another, given by Wolfenstein, ${ }^{7}$ is based on the observation that the forward amplitude for $\bar{v}_{e}-e$ scattering below the $W$ pole in the $s$ channel must have a definite sign; and the third is based on the facts that, in the Glashow-Weinberg-Salam model, the $W$-exchange diagram has the opposite sign to the $Z^{0}$-exchange diagram, and that $Z^{0}$ exchange gives rise to an attractive force between $v_{e}$ and $e$ (Ref. 8). As emphasized by Langacker, the positive sign means that MSW enhancement will now occur when the dominant component of $v_{e}$ is the lighter of the two neutrinos.

As the neutrino travels through the Sun, the density of electrons, $N_{e}$, changes and hence the matrix element $A$ in (3.1) is a function of time. If we eliminate $v_{\mu}$ from the coupled equations of (3.1) and take out a time-dependent phase factor from $v_{e}$,

$$
v_{e}(t)=p(t) \exp \left(-i \int_{0}^{t} A\left(t^{\prime}\right) d t^{\prime}\right),
$$

we find that $p(t)$ obeys the second-order equation

$$
\ddot{p}-i(A-D) \dot{p}+B^{2} p=0 \text {. }
$$

The probability that a neutrino born as a $v_{e}$ at $t=0$ remains a $v_{e}$ at time $t$ is given by

$$
P\left(v_{e} \rightarrow v_{e} ; t\right)=|p(t)|^{2} .
$$

The relationship between the effective mixing angle for oscillations in matter, $\sin ^{2} 2 \theta_{m}$, and the intrinsic mixing angle $\sin ^{2} 2 \theta$ can be written as

$$
\begin{aligned}
& \sin ^{2} 2 \theta_{m}=\frac{\sin ^{2} 2 \theta}{\left[\sin ^{2} 2 \theta+\left(L / L_{0}-\cos 2 \theta\right)^{2}\right]}, \\
& L \equiv 4 \pi E / \Delta m^{2}, L_{0} \equiv 2 \pi /\left(\sqrt{2} G_{F} N_{e}\right) .
\end{aligned}
$$

Obviously the MSW enhancement occurs at $L / L_{0}$ $=\cos 2 \theta$ and its full width at half maximum is given by

$$
2 \Delta\left(L / L_{0}\right)=2 \sin 2 \theta \text {. }
$$

For very small intrinsic mixing angles $\left(\sin ^{2} 2 \theta\right.$ $\simeq 10^{-3}-10^{-1}$ ) this is a very narrow region, and outside it $\sin ^{2} 2 \theta_{m}$ is very small. Thus we can adopt a simple model for small mixing angles in which a neutrino travels from the core of the Sun to the narrow region around the enhancement without oscillating, undergoes maximal oscillations inside this region, and emerges from it as some admixture of $v_{e}$ and $v_{\mu}$ which remains essentially unchanged all the way to Earth.

In terms of the electron density $\rho_{e}$, measured in units of Avogadro's number, the ratio $L / L_{0}$ is given by

$$
L / L_{0}=10^{-7}\left(E / \Delta m^{2}\right) \sqrt{2} \rho_{e}
$$

and the density at enhancement is

$$
\left(\rho_{e}\right)_{0}=\frac{10^{7}}{\sqrt{2}}\left(\Delta m^{2} / E\right) \cos 2 \theta
$$

For fixed $E / \Delta m^{2}$, the change in density across the MSW-enhancement region is from (3.8):

$$
\begin{aligned}
2 \Delta \rho_{e} & =\sqrt{2} \times 10^{7}\left(\Delta m^{2} / E\right) \sin 2 \theta \\
& =2\left(\rho_{e}\right)_{0} \tan 2 \theta .
\end{aligned}
$$

The corresponding spatial extent of the region is therefore

$$
2 \Delta R_{0} \equiv 2 \Delta t_{0}=2\left[\Delta \rho_{e} /\left(d \rho_{e} / d R\right)\right]_{0} \text {. }
$$

We now examine the model in which the neutrino oscillates in the region

$$
\left(R_{0}-\Delta R_{0}, R_{0}+\Delta R_{0}\right) \equiv\left(t_{0}-\Delta t_{0}, t_{0}+\Delta t_{0}\right)
$$

and nowhere else; $R_{0}=t_{0}$ is the location of the MSW enhancement.

An equivalent way of writing the enhancement condition $\left(L / L_{0}=\cos 2 \theta\right)$ is $A-D=0$ [see (3.1) and (3.2)]: therefore the probability amplitude $p(t)$ of (3.5) obeys a simple oscillatory equation $\ddot{p}+B^{2} p=0$ in the neighborhood of the enhancement. If we assume that just before it hits the enhancement region $p=e^{i \alpha}$, then from continuity across the enhancement region boundary we find that

$$
\begin{aligned}
p(t)=e^{i \alpha} \cos B\left(t-t_{0}+\Delta t_{0}\right) & \\
& \left(t_{0}-\Delta t_{0} \leq t \leq t_{0}+\Delta t_{0}\right) .
\end{aligned}
$$

Thus $p(t)$ emerges from the enhancement region with the value

$$
p\left(t_{0}+\Delta t_{0}\right)=e^{i \alpha} \cos \left(2 B \Delta t_{0}\right)
$$

and the probability for its being an electron neutrino at Earth is

$$
P\left(v_{e} \rightarrow v_{e}\right)_{\text {Earth }} \equiv \cos ^{2}\left(\frac{\sin ^{2} 2 \theta}{h_{0}} \frac{\Delta m^{2}}{2 E}\right),
$$

where $h_{0}$ is the scale height of the solar density distribution at enhancement:

$$
h_{0} \equiv-\left(\frac{1}{\rho_{e}} \frac{d \rho_{e}}{d R}\right)_{0} .
$$

The essential feature of this model, and the one that determines its domain of validity, is that the distance $2 \Delta R_{0}$ over which maximal mixing occurs $\left(\sin ^{2} 2 \theta_{m} \simeq 1\right)$ is assumed to be small compared with the oscillation length at the point of enhancement:

$$
2 \Delta R_{0} \ll L_{m} \equiv 4 \pi E /\left(\Delta m^{2} \sin 2 \theta\right)=\pi / B .
$$

Thus the argument of the cosine function in (3.14) and (3.15) is always less than $\pi$, and the change in the nature of the incident neutrino takes place in some fractional part of the maximally oscillating wave. In other words, the region sensitive to MSW enhancement is so small that it locks in only a part of the wave.

This is essentially the reverse of the adiabatic approximation, ${ }^{9}$ in which the region of large mixing angles $\sin ^{2} 2 \theta_{m}$ must be large compared with the oscillation length $L_{m}$ at the point of enhancement. This condition makes it possible for the eigenvectors of the "Hamiltonian" (or effective mass) matrix (3.1) and (3.2) to rotate 

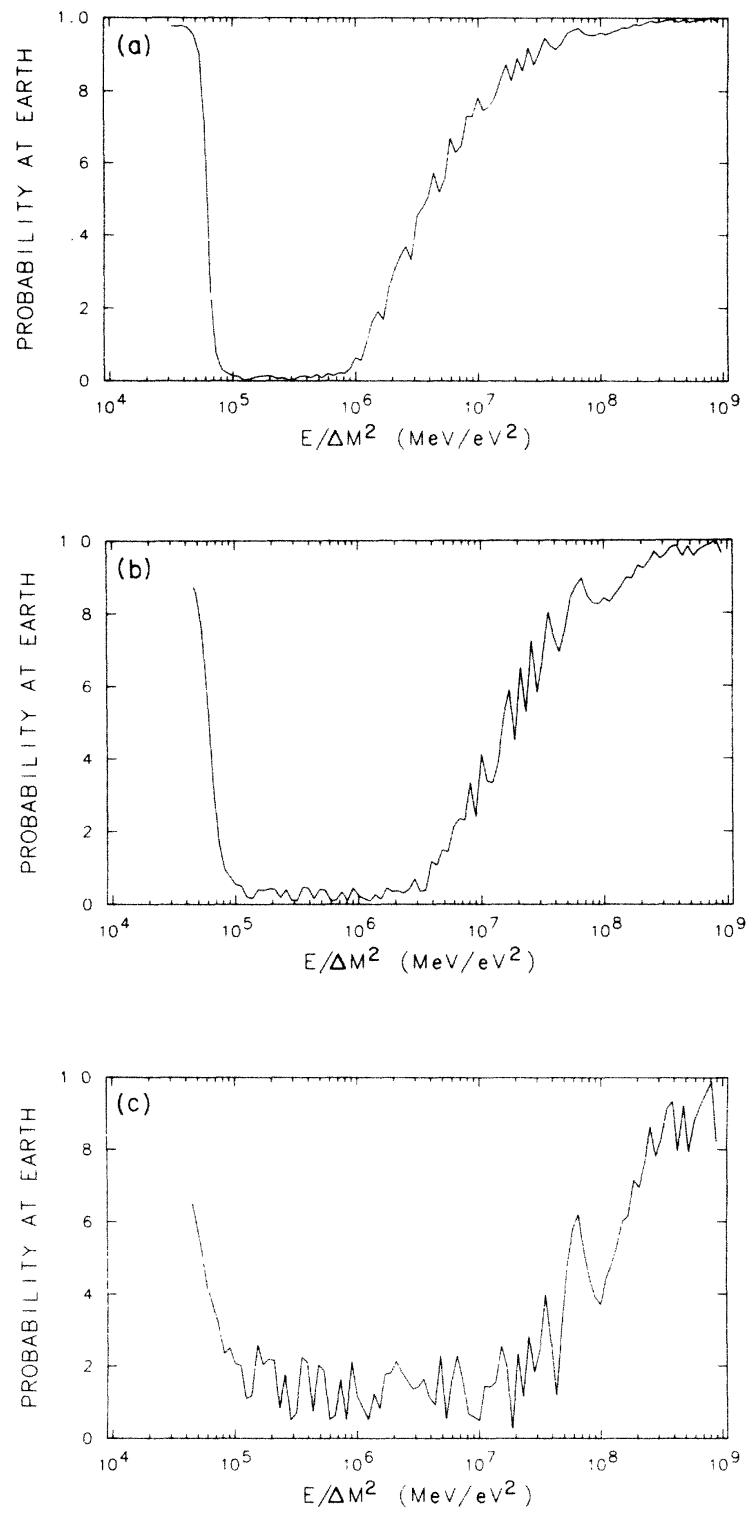

$(M O D|F| E D)$

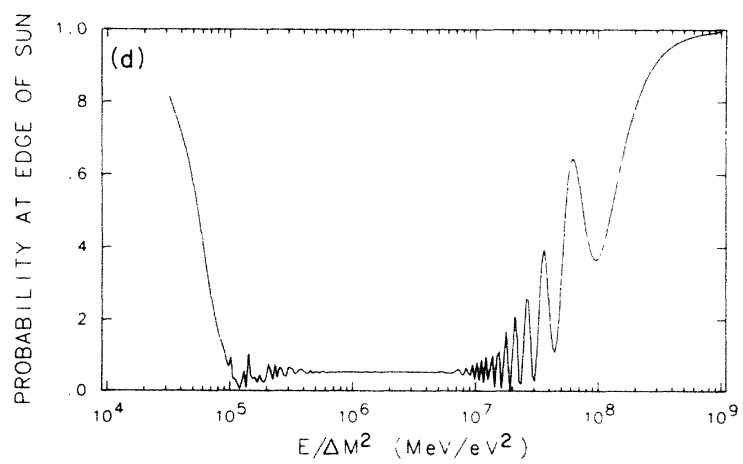

FIG. 1. Probability that a single-electron neutrino created at the center of the Sun arrives at Earth versus $E / \Delta m^{2}$ for a given $\sin ^{2} 2 \theta$. (a) $\sin ^{2} 2 \theta=0.01$; (b) 0.04 ; (c) and (d) 0.20 . (d) is modified: probability at edge of the Sun and more $E / \Delta m^{2}$ points help smooth out the plot. slowly as the neutrino moves through the enhancement region; consequently an eigenvector associated with the larger (smaller) of the eigenvalues at one point in the path of the neutrino maintains its association with the larger (smaller) eigenvalue at another point.

In terms of $\sin ^{2} 2 \theta$ and $E / \Delta m^{2}$, the criterion for validity of our slab model is that

$$
\sin ^{2} 2 \theta /\left(E / \Delta m^{2}\right)<2 \pi h_{0} .
$$

For a fixed, small value of $\sin ^{2} 2 \theta$, the probability for $v_{e} \Longrightarrow v_{e}$ at Earth (3.15) starts out at 1, goes down to zero, and climbs steadily back to 1 as $E / \Delta m^{2}$ increases through the range of values consistent with (3.18). The scale for this behavior is set by $\sin ^{2} 2 \theta$ : the smaller $\sin ^{2} 2 \theta$, the smaller the region in $E / \Delta m^{2}$ for which the probability remains small. These qualitative features are well illustrated by the results of computer calculations shown in Fig. 1, especially for the regime in which the probability climbs from small values back to large ones. Earlier parts of the curve are better described by the adiabatic approximation.

\section{APPLICATIONS OF THE SLAB MODEL}

Another aspect of the slab model concerns the relation between $\sin ^{2} 2 \theta$ and $\Delta m^{2}$ for a fixed suppression. If the scale height $h_{0}$ is a constant, as happens for the exponential density distribution found in a large part of the Sun beyond the core (see Fig. 7), then for a fixed value of $P\left(v_{e} \rightarrow v_{e}\right)$, we must have, from (3.15), that

$$
\left(\sin ^{2} 2 \theta\right)\left(\Delta m^{2}\right)=\mathrm{const}
$$

or

$$
\log _{10}\left(\sin ^{2} 2 \theta\right)+\log _{10}\left(\Delta m^{2}\right)=\text { const } .
$$

Furthermore this property survives when we integrate the product $P\left(v_{e} \rightarrow v_{e}\right) \times$ flux $\times$ capture cross section over the energy range of ${ }^{8} \mathrm{~B}$ neutrinos. By a simple change of variables we can show in this case that the quantity fixed by a given total suppression of the ${ }^{8} \mathbf{B}$ capture rate [in other words, the analog of the argument of the cosine function in (3.15)] is

$$
Q=\left(\frac{\sin ^{2} 2 \theta}{h_{0}} \frac{\Delta m^{2}}{E_{\max }}\right),
$$

where $E_{\max }$ is the maximum energy of the ${ }^{8} \mathbf{B}$ neutrinos. Furthermore, $Q$ has the general property that as the integral of $P\left(v_{e} \rightarrow v_{e} ; E\right)$ increases, so the value of $Q$ must decrease.

Given the scale height of the density distribution in the body of the Sun, we can use (4.2) to determine the constant appearing on the right-hand side of (4.1). If we express the density at $R$ as an exponential function

$$
\rho_{e}(R)=\rho_{e}(0) \exp (-R / \beta),
$$

where $R$ and $\beta$ are measured in meters, then we find from Fig. 7 that

$$
\beta \simeq R_{\text {Sun }} / 10 \simeq 7 \times 10^{7} \mathrm{~m} .
$$

The scale height is simply the inverse of $\beta$, and so 


$$
h_{0}=\left(7 \times 10^{7}\right)^{-1} m^{-1} \simeq 3 \times 10^{-15} \mathrm{eV} .
$$

It then follows from (4.2) that

$$
\begin{aligned}
\log _{10}\left(\sin ^{2} 2 \theta\right)+\log _{10}\left(\Delta m^{2}(\mathrm{eV})^{2}\right)= & \log _{10} Q+\log _{10} h_{0} \\
& +\log _{10}\left(E_{\max }(\mathrm{eV})\right) \\
= & \log _{10} Q-7.4 .
\end{aligned}
$$

Since $Q$ is a number of order 1 for suppression factors in the range $2-4$, we obtain

$$
\log _{10}\left(\sin ^{2} 2 \theta\right)+\log _{10}\left(\Delta m^{2}\right) \simeq-7.4 \text {. }
$$

Considering the crudeness of our model, the agreement between (4.7) and the second solution in Fig. 5(a) is extremely good. Because we neglect energy spreading, spatial spreading, and multineutrino types (all of which are included in Fig. 5), we can expect some discrepancies.

We also note that as $P\left(v_{e} \rightarrow v_{e}\right)$ increases (i.e., the suppression factor decreases), $Q$ decreases and so the constant on the right-hand side of (4.6) decreases. Thus the second line in Fig. 5 should move down the page as the suppression factor decreases, and up the page as it increases. This is exactly what happens in the computer calculations.

From (3.18), the criterion for the validity of the slab model for the exponential density distribution is

$$
\left(\sin ^{2} 2 \theta\right)\left(\Delta m^{2}\right)<2 \pi h_{0} E(\mathrm{eV}) \simeq 10^{-6.7} .
$$

The range of parameters on the second line are well within this limit.

As has been emphasized by Bethe, ${ }^{9}$ the first line solution of Fig. 5 corresponds to the adiabatic approximation, which is valid when the inequality in Eq. (3.18) is reversed. This approximation accounts for the earlier (in $\left.E / \Delta m^{2}\right)$ parts of the curves of Fig. 1 in which the proba- bility for an original $v_{e}$ to remain a $v_{e}$ at Earth starts out close to unity, falls rapidly, and then remains close to zero. Since the region of rapid fall occurs close to $E / \Delta m^{2} \approx 10^{5}$ for a large range of values for $\sin ^{2} 2 \theta$, the solution is approximately constant as a function of mixing angle. The value of $\Delta m^{2}\left(\approx 10^{-4} \mathrm{eV}^{2}\right)$ indicates that the enhancement occurs in the core region of the Sun.

\section{COMPUTER CALCULATIONS}

Equation (3.5) plus the initial conditions $\left\langle v_{e}(0) \mid v_{e}(0)\right\rangle=1$ and $\left\langle v_{\mu}(0) \mid v_{\mu}(0)\right\rangle=0$ form the basis of our computations. It is convenient to represent $p$ as $w+i s$. Equation (3.5) then becomes two coupled secondorder differential equations in $w$ and $s$. The above initial conditions can be shown to be equivalent to $w(0)=1$ and $s(0)=\dot{w}(0)=\dot{s}(0)=0$.

We use Runge-Kutta techniques for two coupled second-order differential equations to solve for $P_{\text {edge }}$. We use the following parameters:

$$
\begin{aligned}
x & \equiv R / R_{\text {Sun }}\left(R_{\text {Sun }} \cong 6.96 \times 10^{5} \mathrm{~km}\right), \\
L & \equiv 4 \pi E / \Delta m^{2} \\
& =2.5 \times 10^{-3} E / \Delta m^{2}\left[\mathrm{MeV} /(\mathrm{eV})^{2}\right] \mathrm{km}, \\
L_{0} & \equiv 2 \pi /\left(\sqrt{2} G_{F} N_{e}\right) \cong 1.77 \times 10^{4} / \rho_{e}(x) \mathrm{km}, \\
\Delta x & =0.00001,
\end{aligned}
$$

where $\rho_{e}(x)$ is the hydrogen/helium corrected electronic density ${ }^{10}$ in units of Avogadro's number (see Fig. 7).

Once we know $w, \dot{w}, s$, and $\dot{s}$ at the edge of the Sun ( $P_{\text {edge }} \equiv w^{*} w+s^{*} s$ ), the probability of Earth, $P_{\text {Earth }}$, can be computed making use of the equation for vacuum-only oscillations; ${ }^{11}$ we obtain

$$
\begin{aligned}
P_{\text {Earth }}= & \sin ^{2} 2 \theta \sin ^{2}\left(\pi R_{e e} / L\right)\left(1-2 P_{\text {edge }}\right)+P_{\text {edge }}+\sin 2 \theta \cos 2 \theta \sin ^{2}\left(\pi R_{e e} / L\right)(2 / B)(w \dot{s}-s \dot{w}) \\
& +\sin 2 \theta \sin \left(\pi R_{e e} / L\right) \cos \left(\pi R_{e e} / L\right)(2 / B)(w \dot{w}+s \dot{s}),
\end{aligned}
$$

where $R_{e e}$ is the distance from the edge of the Sun to Earth ( $\cong 1.5 \times 10^{8} \mathrm{~km}$ ) and $L$ is as defined in (5.1). The last two terms in (5.2) are interference terms which can be constructive or destructive and help explain why the vacuumlike oscillations in Fig. 2 are not bounded by $\sin ^{2} 2 \theta$.

The energy spectrum for ${ }^{8} \mathbf{B}$ neutrinos was taken from Bahcall, ${ }^{12}$ that for ${ }^{7} \mathrm{Be}$ from Bahcall et al., ${ }^{4}$ and the latest $p p$ spectrum was personally given to us by Bahcall. The most recent normalizations were used $\left(4.0 \times 10^{6} \mathrm{no} . / \mathrm{cm}^{2} /\right.$ sec for ${ }^{8} \mathrm{~B}, 3.2 \times 10^{9}$ for both ${ }^{7} \mathrm{Be}$ lines combined, and $6.1 \times 10^{10}$ for $p p$ ) (Ref. 13). Chlorine cross sections were taken from Bahcall ${ }^{14}$ and gallium cross sections were taken from Bahcall. ${ }^{12}$

We wrote a series of programs of increasing complexity using the data described above. The simplest program computes $P\left(v_{e} \rightarrow v_{e}\right)$ at Earth as a function of $E / \Delta m^{2}$ for a given $\sin ^{2} 2 \theta$ assuming that a single electron neutrino is produced at the center of the Sun; typical results are shown in Figs. 1(a), 1(b), and 1(c). The roughness of the oscillations about the envelope are an artifact of the plot, but the oscillations themselves are real and can be smoothed out by taking finer steps in $E / \Delta m^{2}$, as illustrated in Fig. 1(d).

The next program follows the probability $P\left(v_{e} \rightarrow v_{e}\right)$ for an electron neutrino as it travels through the Sun with a given mixing angle and oscillation length. Figure 2(a) beautifully displays the regions of suppression, amplification, and vacuum-only oscillations as described by $\mathrm{Mi}$ kheyev and Smirnov; Fig. 2(b) shows a similar graph for a smaller oscillation length, demonstrating a larger suppression of $v_{e}$. Figures 2(c) and 2(d) show examples of "double enhancement." Neutrinos produced behind the center of the Sun can sometimes undergo enhancement twice due to the symmetric nature of the Sun. However, the derivative of the density switches sign traversing the center. This can lead to further suppression, Fig. 2(c), or raise the probability back up near one, Fig. 2(d), depending on the phase of the wave arriving at the second region of enhancement (thereby dependent on $E / \Delta m^{2}$ ).

Subsequent programs take into account the dependence 

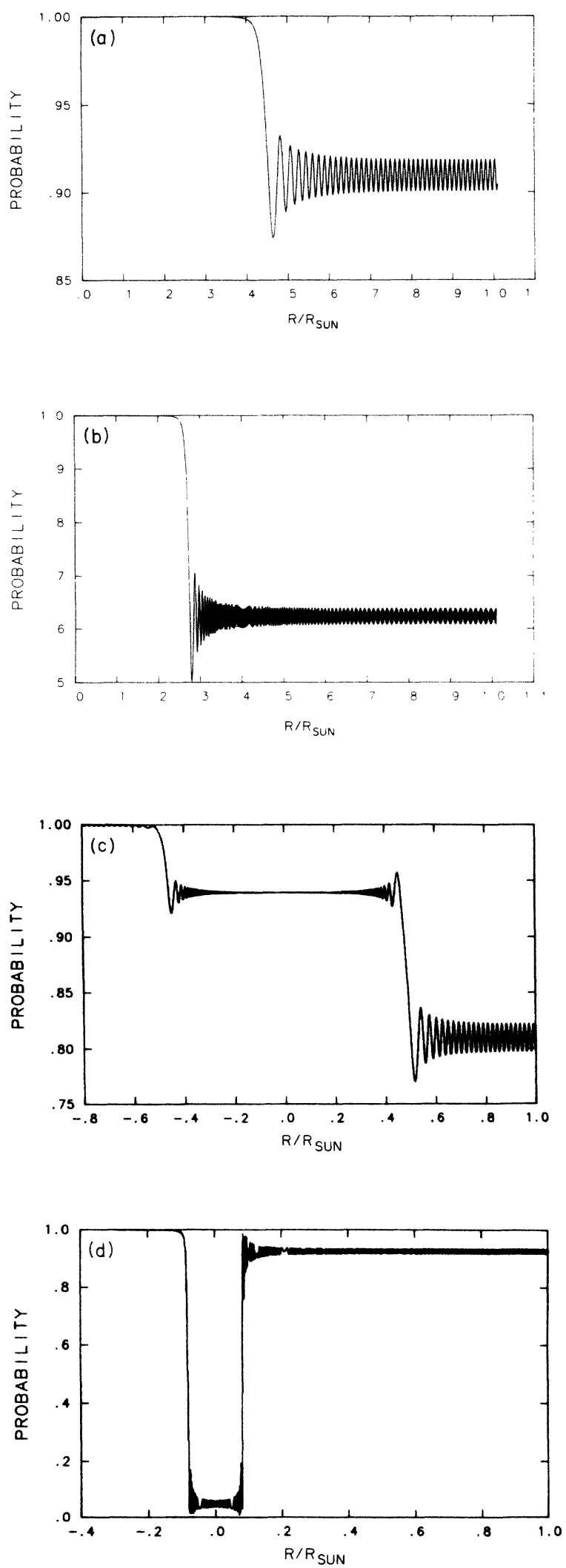

FIG. 2. Probability of a single-electron neutrino created at the center of the Sun at each point in the Sun for a given $\sin ^{2} 2 \theta$ and $E / \Delta m^{2}$. All plots are for $\sin ^{2} 2 \theta=0.001$. (a) $E / \Delta m^{2}$ $=3 \times 10^{6} \mathrm{MeV} / \mathrm{eV}^{2}$; (b) $6 \times 10^{5}$; (c) "double enhancement" $E / \Delta m^{2}=5 \times 10^{6} \mathrm{MeV} / \mathrm{eV}^{2}$; (d) "double," $1 \times 10^{5}$. of $P_{\text {Earth }}$ on where the neutrinos are produced. ${ }^{8} \mathrm{~B}$ neutrinos are produced predominantly at the center of the Sun, but $p p$ neutrinos can be produced as far out as one quarter of the solar radius. For comparison, Fig. 3(a) shows the modified results for $\boldsymbol{P}_{\text {Earth }}$ with the same parameters as were used to generate Fig. 1(a); note that the region of suppression is now slightly smaller than before. A greater shrinking of this region occurs for $p p$ neutrinos as can be seen from a comparison of Figs. 3(b) and 1(a). The method by which spatial dependence is taken into account is described in Appendix A.

The most comprehensive programs allow for the capture cross sections (see Appendix B) and the energy spectra of the ${ }^{8} \mathrm{~B}$ and $p p$ neutrinos, and they yield capture rates as functions of $\Delta m^{2}$ for specific values of $\sin ^{2} 2 \theta$. Typical results for $\sin ^{2} 2 \theta=0.01$ are shown in Fig. 4 in which an averaging process (see Appendix B) has been used to smooth out the curves. Our most important results are contained in Fig. 5, which determines the values of $\sin ^{2} 2 \theta$ and $\Delta m^{2}$ for a given reduction of the ${ }^{8} \mathrm{~B}$ capture rate; Table $I$, which gives the predicted capture rates for the ${ }^{71} \mathrm{Ga}$ experiment for the parameters shown in Fig. 5(a); and Fig. 6, which shows the modified spectra of ${ }^{8} \mathrm{~B}$ neutrinos at Earth corresponding to these parameters. (Figure 6 is presented in numerical form in Table II.) We

\section{(8B)}
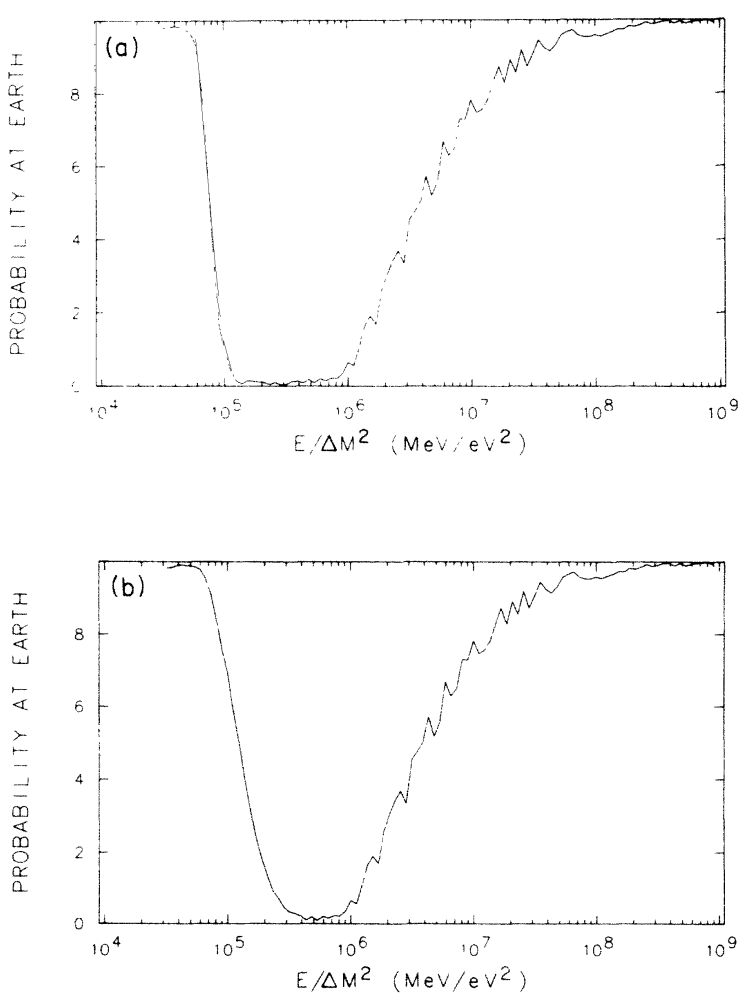

FIG. 3. Similar to Fig. 1 but with spatial spreading (see Appendix A) taken into account. Both plots are for $\sin ^{2} 2 \theta=0.01$. (a) ${ }^{8} \mathrm{~B}$ neutrinos; (b) $p p$ neutrinos. Not depicted: ${ }^{7} \mathrm{Be}$ neutrinos whose effect lies somewhere in between. All subsequent programs take spatial spreading into account. 


\section{$(8 B$ ON $37 \mathrm{CL})$}

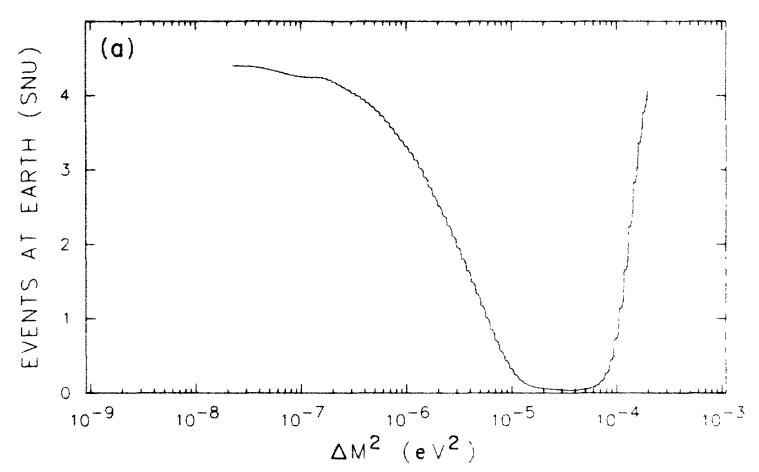

(8B PLUS 7BE ON 37CL)

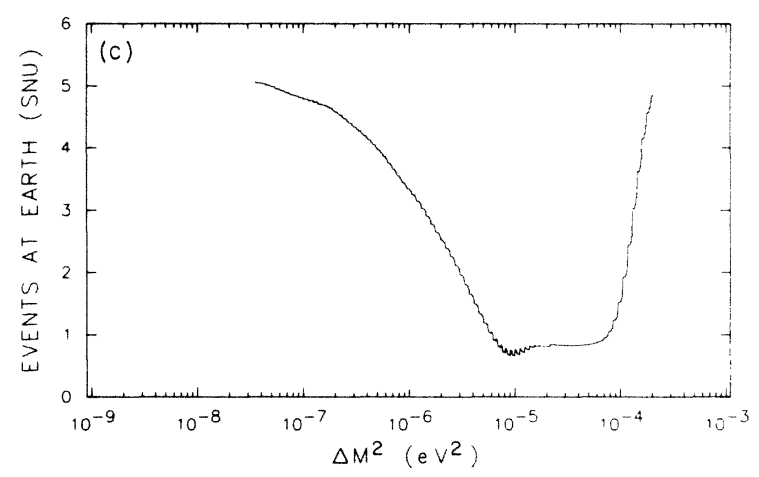

( $7 B E$ ON 71GA)

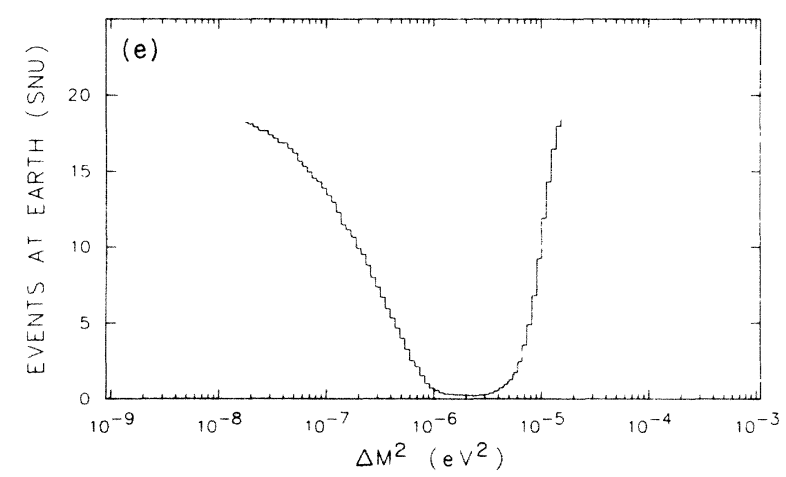

( $7 B E$ ON $37 C L)$

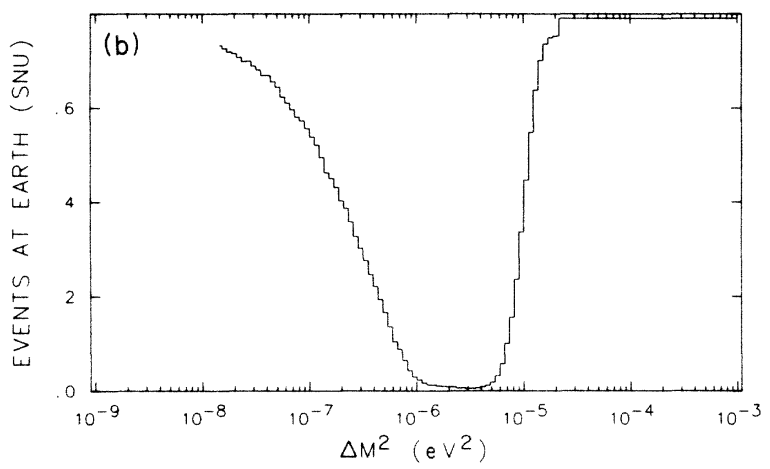

(PP ON 71GA)

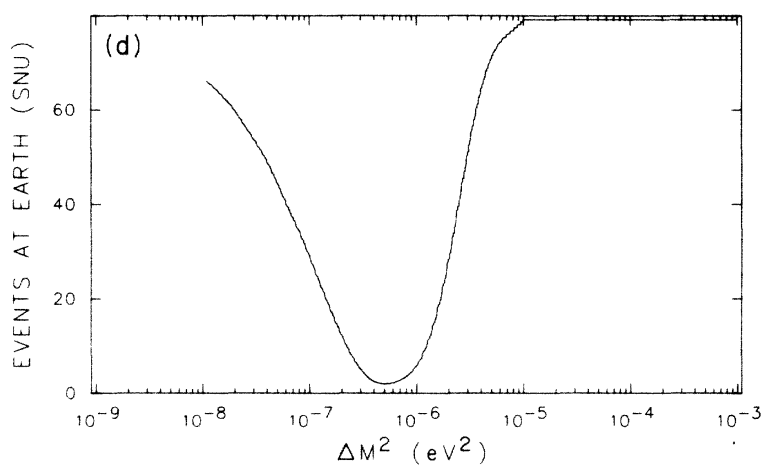

(PP PLUS 7BE ON 71GA)

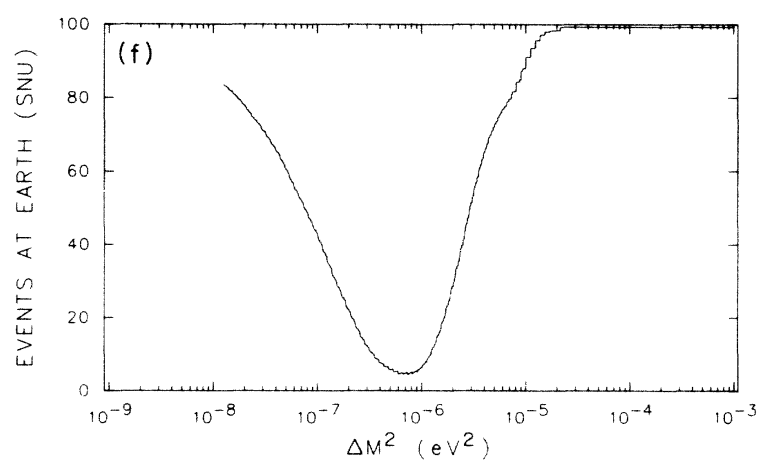

FIG. 4. Energy spectra and detector cross sections were integrated out (see Appendix B) to yield event rates versus $\Delta m^{2}$. 1 SNU $=10^{-36}$ events/scatterer/sec. All plots are for $\sin ^{2} 2 \theta=0.01$. The $100 \%$ (no oscillation) values in SNU are (a) 4.5; (b) 0.83 ; (d) 83.33; (e) 21.18. (c) is the sum of (a) and (b). (f) is the sum of (d) and (e). 
have concentrated on the small angle region, but the programs work with arbitrary mixing angles.

\section{FINAL COMMENTS}

We have shown by extensive computation that, if the anomalously low capture rate for solar neutrinos observed by Davis is due to enhancement of neutrino oscillations in the Sun itself, the Mikheyev-Smirnov-Wolfenstein or MSW effect, then there are two classes of solution for the oscillation parameters in a two-state model. In one case, the enhancement takes place in the core region of the Sun and $\Delta m^{2}$ is roughly constant, with value $\simeq 10^{-4} \mathrm{eV}^{2}$, for a range of values of $\sin ^{2} 2 \theta$ between $10^{-3}$ and $10^{-1}$.

( 8 B PLUS 7BE ON $37 \mathrm{CL}$ )

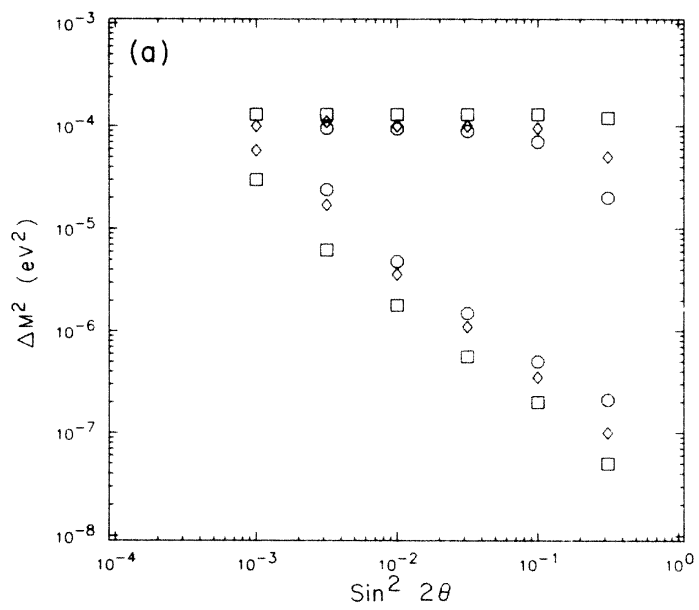

(PP PLUS 7BE ON 71GA)

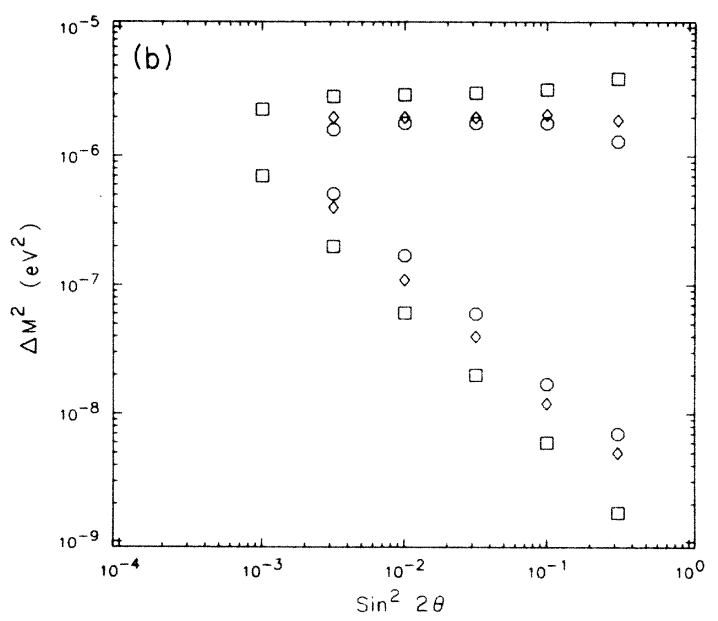

FIG. 5. Programs similar to those that produced Fig. 4 were run for several values of $\sin ^{2} 2 \theta$ : the values of $\Delta m^{2}$ were collected that yield reductions in the event rate by factors $2-4$. (a) ${ }^{8} \mathrm{~B}+{ }^{7} \mathrm{Be}$ in ${ }^{37} \mathrm{Cl}$; (b) $p p+{ }^{7} \mathrm{Be}$ in ${ }^{71} \mathrm{Ga}$. Boxes $=\frac{1}{2}$ of the expected capture rate; diamonds $=\frac{1}{3}$, circles $=\frac{1}{4}$.

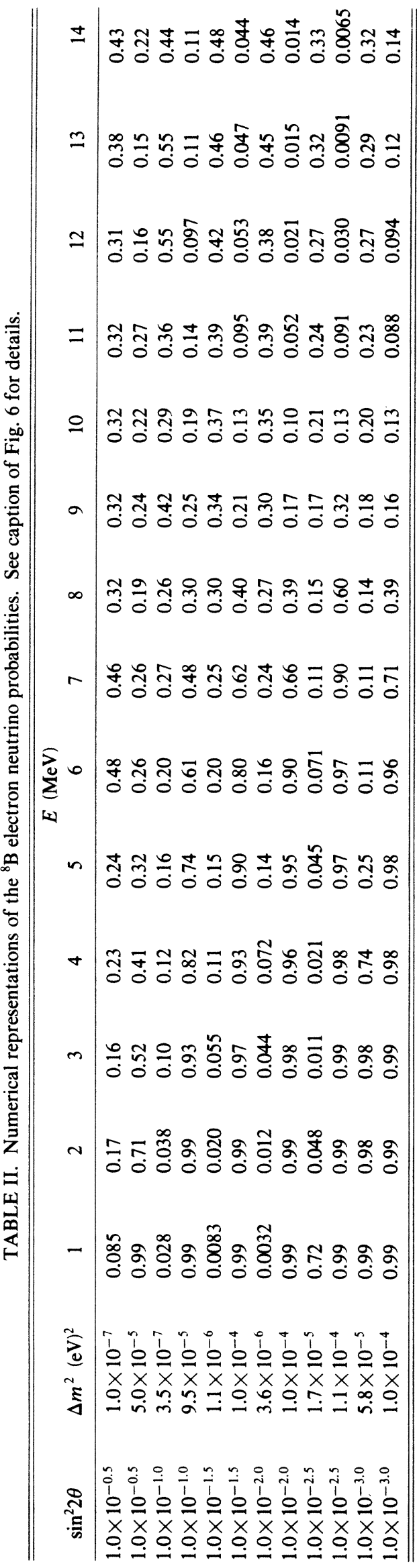



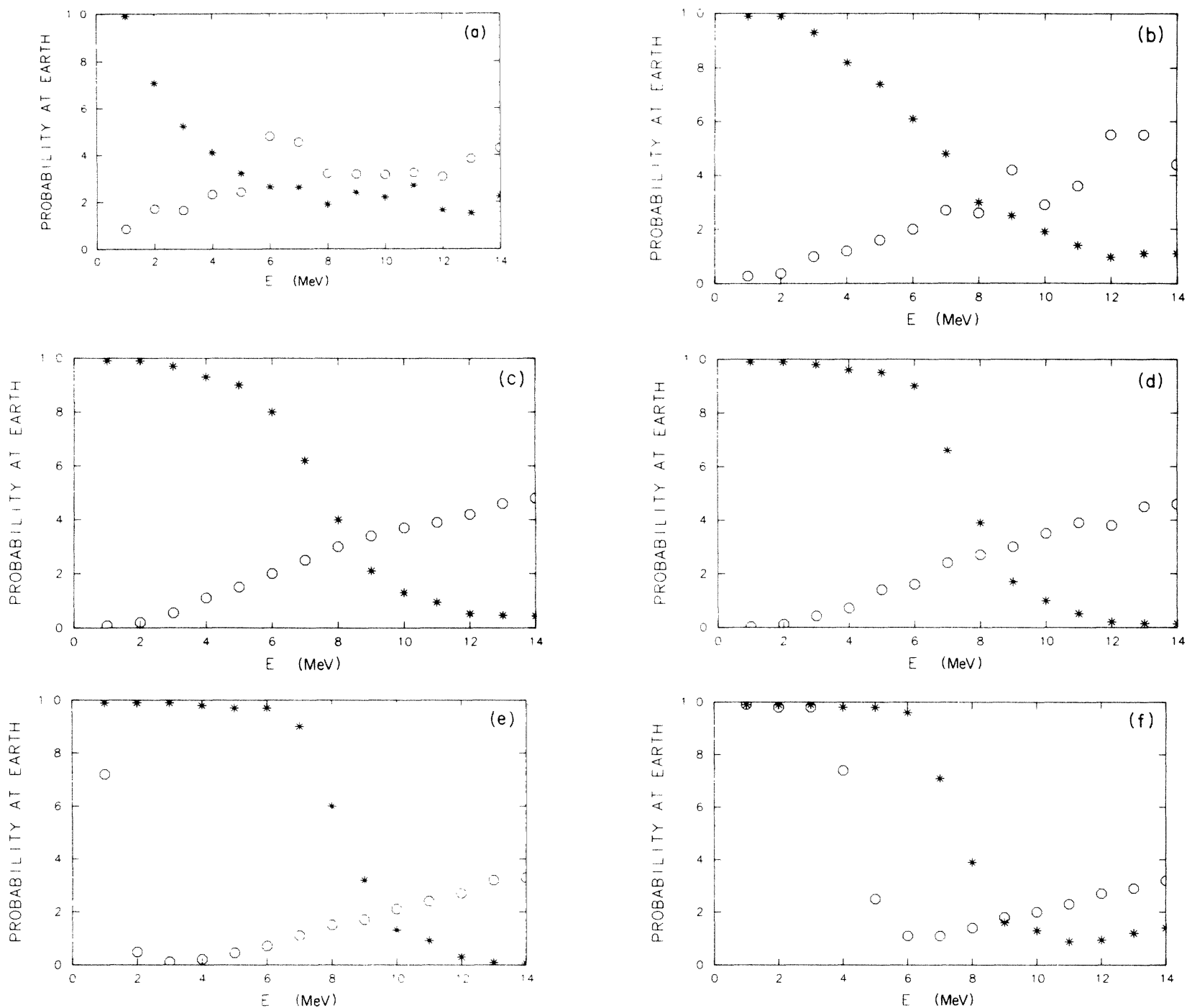

FIG. 6. Values of $\sin ^{2} 2 \theta$ and $\Delta m^{2}$ that yield a factor of 3 reduction in ${ }^{8} \mathrm{~B}+{ }^{7} \mathrm{Be}$ in ${ }^{37} \mathrm{Cl}$ [see Fig. 5(a)] were used to produce plots of probabilities that electron neutrinos arrive at Earth vs $E(\mathrm{MeV}) . N \mathrm{MeV}$ represents results averaged from $N-0.5$ to $N+0.5 \mathrm{MeV}$. (a) $\sin ^{2} 2 \theta=10^{-0.5}, \Delta m^{2}: 0=1.0 \times 10^{-7} \mathrm{eV}^{2}, *=5.0 \times 10^{-5}$; (b) $10^{-1.0}, 3.5 \times 10^{-7}, 9.5 \times 10^{-5}$; (c) $10^{-1.5}, 1.1 \times 10^{-6}, 1.0 \times 10^{-4} ;(\mathrm{d})$ $10^{-2.0}, 3.6 \times 10^{-6}, 1.0 \times 10^{-4}$; (e) $10^{-2.5}, 1.7 \times 10^{-5}, 1.1 \times 10^{-4}$; (f) $10^{-3.0}, 5.8 \times 10^{-5}, 1.0 \times 10^{-4}$.

(See Fig. 7.) In the second class, the enhancement occurs in the main body of the Sun, where the density falls off exponentially, and we find that the product $\left(\sin ^{2} 2 \theta\right)\left(\Delta m^{2}\right) \simeq 10^{-7.5}$. We have also shown that the two classes yield different spectra for the ${ }^{8} \mathrm{~B}$ neutrinos arriving at Earth: in the former case, the low-energy neutrinos $(E \leq 5-7 \mathrm{MeV})$ are unaffected by oscillations, but the high-energy ones are strongly suppressed; whereas in the latter case neutrinos of all energies are suppressed, but most especially those with low or intermediate energies. This contrasting behavior may prove to be a key element in the resolution of the solar neutrino problem.

Our emphasis on small in vacuo mixing angles led us to a simple model in which any change in the nature of the neutrino occurs in a relatively thin slab around the enhancement density. This model works surprisingly well in accounting for the results of our computations, but the criterion for its validity is the reverse of the criterion for
ELECTRONIC DENSITY

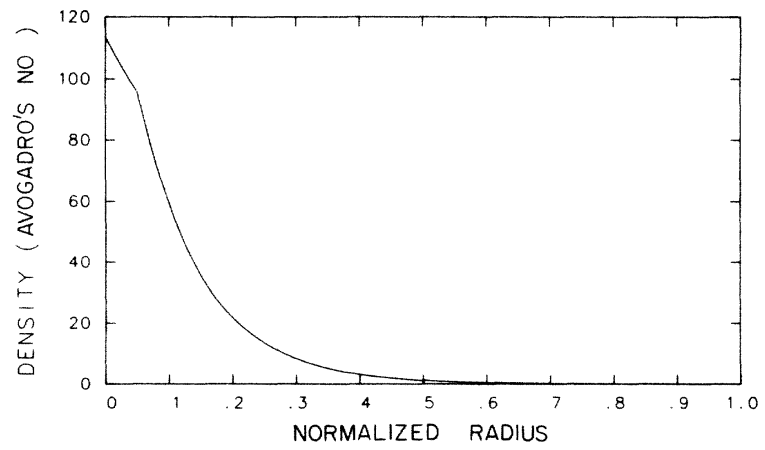

FIG. 7. Solar electronic density in units of Avogadro's number. Generated by combining mass density and hydrogen/ helium mixture plots. Note the slower dropoff in the core than in the body. 
the adiabatic approximation: our model requires the product $\left(\Delta m^{2}\right)\left(\sin ^{2} 2 \theta\right) / E$ to be smaller than a certain number, whereas the adiabatic approximation requires the same product to be greater than another number, roughly equal to the first one. Thus the two approximations complement each other.

\section{ACKNOWLEDGMENTS}

The authors would like to thank Joe Weneser who introduced them to the work of Mikheyev and Smirnov. Ray Davis, Sid Bludman, and Peter Vogel participated in many stimulating conversations with them. Jim Friar assisted them in determining the absolute sign of the neutrino-electron forward-scattering amplitude, and Lincoln Wolfenstein assisted in understanding the square root of two that appears in the equations for matter oscillations. Art Cox and Russel Kidman provided them with an electronic density profile, and John Bahcall and Bruce Cleveland provided them with access to much information about solar neutrinos. They are also indebted to A. Messiah for an extremely helpful discussion regarding the adiabatic approximation and to $\mathbf{A}$. Smirnov and $\mathbf{B}$. Kayser for discussions on matters of coherence. Wick Haxton and $\mathrm{Al}$ Petschek were helpful in pointing out the existence of the interference terms in Eq. (5.2).

\section{APPENDIX A: SPATIAL DEPENDENCE OF PRODUCTION RATES}

To account for the spatial dependence of production rates-the dependence on where the neutrinos are actually produced in the Sun-we divided the Sun into a semicircular grid (see Fig. 8). By symmetry, this accounts for all the different distances the neutrinos have to travel toward Earth and most importantly the density that the neutrino "sees." The number of semicircles (solid lines) depends on the neutrino type, being fewer for ${ }^{8} \mathrm{~B}$ neutrinos since they are produced near the core, and greater for $p p$ neutrinos which are produced over a larger region. The number of radial lines (dashed lines) was determined by continually increasing their number until the resulting calculations converged. As it turned out-for ${ }^{8} \mathrm{~B},{ }^{7} \mathrm{Be}$, and $p p$ neutrinos-only two dashed lines were needed: $0^{\circ}$ and $180^{\circ}$. It is assumed that the Sun is far enough away for the rays to be considered parallel.

The intersection of solid and dashed lines are the production nodes each assigned a weight $W_{i}$ (normalized to 1) that only depend on the radial direction and were taken from Bahcall et $+a l^{4}$

Let $P_{i}\left(E / \Delta m^{2}\right.$,node $\left.i\right)$ be the probability at Earth calculated from node $i$. The probability we are interested in is

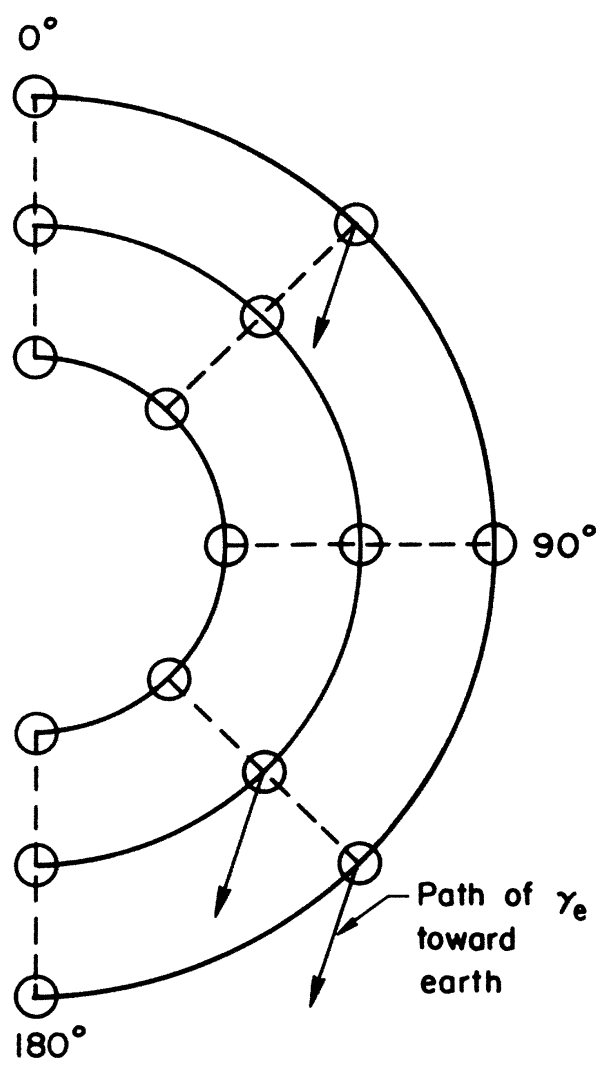

FIG. 8. Typical semicircular grid used to model spatial spreading (see Appendix A).

$$
\begin{gathered}
P_{\text {Earth }}\left(E / \Delta m^{2}\right)=\sum_{i} W_{i} P_{i}\left(E / \Delta m^{2}, \text { node } i\right) \\
\text { APPENDIX B: ENERGY DEPENDENCE } \\
\text { OF PRODUCTION RATES AND DETECTOR } \\
\text { CROSS SECTIONS }
\end{gathered}
$$

When electron neutrinos are produced in the Sun they are produced with a spread in energy which is different for ${ }^{8} \mathrm{~B}$, $p p$, and ${ }^{7} \mathrm{Be}$ neutrinos. We made the spread discrete, choosing various energies $E_{i}$ with fluxes $F_{i}\left(E_{i}\right)$. Detector cross sections, which depend on energy and detector type, were also made discrete: $\sigma_{i}\left(E_{i}\right)$.

From the probability that an electron neutrino reaches Earth versus $E / \Delta m^{2}, P\left(E / \Delta m^{2}\right)$, which is known from our earlier programs, we can find the number of events/scatterer/sec versus $\Delta m^{2}$ in SNU:

$$
\begin{aligned}
C\left(\Delta m^{2}\right)_{\mathrm{SNU}} & =\text { const } \times \sum_{x} \sum_{E} F_{x}(x) F_{E}(E) \sigma_{E}(E) P_{\mathrm{Earth}}\left(E / \Delta m^{2}, x\right) \\
& =\text { const } \sum_{E} F_{E}(E) \sigma_{E}(E) \sum_{x} F_{x}(x) P_{\mathrm{Earth}}\left(E / \Delta m^{2}, x\right) \\
& =\text { const } \sum_{E} F_{E}(E) \sigma_{E}(E) P\left(E / \Delta m^{2}\right) .
\end{aligned}
$$


The sums over $x$ are spatial sums over the production nodes (see Appendix A) and the sums over $E$ are over the discrete energies $E$.

For a given $\Delta m^{2}$, the program "grabs" the appropriate $P\left(E / \Delta m^{2}\right)$ from the output of our earlier calculations for each $E / \Delta m^{2}$. Actually, we average $P\left(E / \Delta m^{2}\right)$ over a small neighborhood to smooth out the graphs. More advanced analysis could include the standard deviation of the neighborhood and other sources of deviation. We did not bother to do so.

\section{APPENDIX C: COHERENCE OF WAVE PACKETS}

Throughout this work we have tacitly assumed that the two in vacuo mass eigenstates within the initial neutrino wave packet remain coherent during the enhancement process. ${ }^{15}$ As discussed by Kayser, ${ }^{16}$ the length $l_{c}$ over which the neutrinos remain coherent is related to the length $l_{p}$ over which they are produced by

$$
l_{c} \simeq 2\left(\frac{[E(\mathrm{eV})]^{2}}{\Delta m^{2}(\mathrm{eV})^{2}}\right) l_{p} .
$$

If $l_{p}$ is of order of atomic dimension $\left(l_{p} \simeq 10^{-10} \mathrm{~m}\right)$, then for ${ }^{8} \mathbf{B}$ neutrinos $l_{c}$ varies between $\left(\frac{1}{10}\right)$ of a solar radius to about one thousand solar radii as $\Delta m^{2}$ ranges from $10^{-4}$ $\mathrm{eV}^{2}$ to $10^{-8} \mathrm{eV}^{2}$. The corresponding oscillation lengths in vacuo are about one thousand times smaller, and hence one does not expect any serious problems with coherence for ${ }^{8} \mathbf{B}$ neutrinos.
For $p p$ neutrinos the coherence lengths are about 3 orders of magnitude smaller than those for ${ }^{8} \mathbf{B}$ neutrinos, ranging from $100 \mathrm{~km}$ to 1000000 as $\Delta m^{2}$ varies over the range given above. Clearly there could be problems with coherence for the larger $\Delta m^{2}$. In general, $l_{c}$ is significantly longer than the in vacuo oscillation length $L$,

$$
\begin{aligned}
l_{c} / L & \simeq 2 \times 10^{-4} E(\mathrm{eV}) \\
& \simeq 60 \text { for } p p \text { neutrinos }
\end{aligned}
$$

and so the oscillation length for $p p$ neutrinos in matter at a point of enhancement will also be much smaller than $l_{c}$ provided that

$$
\sin 2 \theta \gg 1 /\left[2 \times 10^{-4} E(\mathrm{eV})\right] \simeq \frac{1}{60} .
$$

Whether or not the MSW enhancement will occur depends upon the condition:

$$
[E(\mathrm{MeV})] /\left(\Delta m^{2}(\mathrm{eV})^{2}\right)=\left(10^{7} \cos 2 \theta\right) / \rho_{e} .
$$

For $p p$ neutrinos, $E \simeq 0.3 \mathrm{MeV}$ and the mixing angle for enhancement must be

$$
\cos 2 \theta \simeq 0.3\left(\rho_{e} / 100\right)\left(10^{-5} / \Delta m^{2}\right) .
$$

In a significant range of value of $\rho_{e}$ and $\Delta m^{2}$, the in $v a$ cuo mixing angle must be large. Thus, for small mixing angles there can be no MSW enhancement. Combining this argument and the one in (6.3), we conclude that the question of coherence is not a serious one for small in vacuo mixing angles.
1S. P. Mikheyev and A. Yu. Smirnov, Institute for Nuclear Research of Academy of Sciences of the USSR, 60th Anniversary prosp 7a, Moscow 117 342, USSR (unpublished); Nuovo Cimento 9C, 17 (1986).

${ }^{2}$ L. Wolfenstein, Phys. Rev. D 17, 1 (1978).

${ }^{3} \mathrm{R}$. Davis et al., in Science Underground, proceedings of The Workshop on Science Underground, Los Alamos, New Mexico, edited by M. M. Nieto et al. (AIP Conf. Proc. No. 96) (AIP, New York, 1983).

4J. N. Bahcall et al., Rev. Mod. Phys. 54, 767 (1982).

5V. Barger et al., Phys. Rev. D 22, 2718 (1980); R. R. Lewis, ibid. 21, 663 (1980).

6P. Langacker (private communication).

${ }^{7}$ L. Wolfenstein (private communication).

${ }^{8}$ This argument is based upon the well-known property of the standard model that charged and neutral currents interfere destructively in $v_{e}-e$ scattering [see, for example, B. Kayser et al., Phys. Rev. D 20, 87 (1979)]. The force between $v_{e}$ and $e$ generated by $Z_{0}$ is attractive as long as $\sin ^{2} \theta_{W}<\frac{1}{4}$, and hence, as pointed out to us by Friar, the corresponding forward-scattering amplitude is positive. It follows that the forward amplitude from $W$ exchange must be negative.

${ }^{9}$ H. A. Bethe, Phys. Rev. Lett. 56, 1305 (1986). Illuminating discussions of the adiabatic approximation have also been given by A. Messiah and A. Smirnov, in The Workshop on Massive Neutrinos in Physics and Astrophysics, XXI Rencontres de Moriond, Tignes, France, 1986 (to be published in the proceedings, edited by J. Tran Thanh Van). See also V. Barger, R. J. N. Phillips, and K. Whisnant, following paper, Phys. Rev. D 34, 980 (1986).

${ }^{10} \mathrm{~A}$. Cox and R. Kidman, generated by their solar-model computer code at T-6, Los Alamos National Laboratory, Los Alamos, NM 87545.

${ }^{11}$ S. M. Bilenky and B. Pontevorvo, Lett. Nuovo Cimento 17, 569 (1976).

12J. N. Bahcall, in Proceedings of the Neutrino Mass Miniconference, Telemark, Wisconsin, 1980, edited by V. Barger and D. Cline (University of Wisconsin, Madison, 1981).

${ }^{13}$ J. N. Bahcall et al., Astrophys. J. 292, No. 2, Part 2, (1985).

14J. N. Bahcall, Rev. Mod. Phys. 50, 1 (1978).

${ }^{15}$ A. Smirnov (private communication).

${ }^{16}$ B. Kayser, Phys. Rev. D 24, 110 (1981). 